\title{
EMBEDDED-SIGNAL DESIGN FOR CHANNEL PARAMETER ESTIMATION PART II: QUANTIZATION EMBEDDING
}

\author{
Pierre Moulin \\ University of Illinois \\ Beckman Inst., Coord. Sci. Lab \& ECE Dept. \\ 405 N. Mathews Ave., Urbana, IL 61801 \\ moulin@ifp.uiuc.edu
}

\begin{abstract}
This is Part II of a paper devoted to the problem of waveform design for identification of communication channel parameters, where the waveform is to be embedded in a host signal. We study a quantization-based embedding method and a two-step parameter estimator. The first step is a decoding problem, and the second step is a nonlinear leastsquares minimization problem. Estimation performance is studied in an asymptotic regime. Under some conditions, the estimator does as well as one that knows the host signal.
\end{abstract}

\section{INTRODUCTION}

Part I of this paper [1] studies a framework for channel parameter estimation based on embedded signals. Linear embedding techniques are considered, and it is shown that subspace projection methods are capable of host-signal rejection under a certain uniform orthogonality condition for the family of channels considered. An important disadvantage of these methods however is their apparent vulnerability in security applications (e.g, watermarking and covert communications).

The embedding system studied here is based on randomized Quantization Index Modulation (QIM) [2, 3], which is an information-theoretic binning scheme. It is known that QIM possesses host-signal rejection properties for coding applications [2, 3] and detection applications [4]. We show here that these properties extend to the problem of estimating a parameter in a compact set $\Theta$.

\section{MATHEMATICAL MODEL}

Consider a linear communication channel with input $x \in$ $\mathbb{R}^{n}$ and output

$$
y=G_{\theta}(x+w), \quad \theta \in \Theta, \quad y \in \mathbb{R}^{n},
$$

where $w \in \mathbb{R}^{n}$ is i.i.d. Gaussian noise with mean zero and variance $\sigma_{w}^{2}$, and $\mathcal{G}=\left\{G_{\theta}, \theta \in \Theta\right\}$ is a given family of $n \times$

\footnotetext{
Work supported by NSF under Grant CCR 00-81268.
}

$n$ invertible matrices. ${ }^{1}$ The parameter set $\Theta$ is a compact subset of $\mathbb{R}^{m}$; the parameter $\theta$ is to be estimated from the channel output. Examples of (1) include delays, scaling, time-varying delay, amplitude modulation, filtering, etc.

The channel input signal $x$ must be "close" to a host signal $s \in \mathbb{R}^{n}$, modeled as a zero-mean random vector with full-rank covariance matrix $R_{s}$. The channel input $x$ is of the form $x=\Phi(s, p)$, where $p$ is a pseudorandom sequence, and $\Phi$ satisfies the expected squared-error distortion constraint

$$
\mathbb{E}_{P S}\|X-S\|^{2} \leq n D_{e}
$$

Here $\mathbb{E}_{P S}$ denotes expectation with respect to the distribution of $p$ and $s$, and $D_{e}$ denotes embedding distortion per sample. The estimator of $\theta$ knows $p$, but not $s$. Given this model, we want to design the input signal $x$ subject to the distortion constraint (2) so as to accurately estimate $\theta$.

For simplicity of the notation we shall henceforth assume that $\Theta \subset \mathbb{R}$. We also define ${ }^{2} H_{\theta}=G_{\theta}^{-1}, \dot{G}_{\theta}=$ $\frac{d}{d \theta} G_{\theta}, Q_{\theta}=G_{\theta} G_{\theta}^{T}, \dot{Q}_{\theta}=\frac{d}{d \theta} Q_{\theta}$, and $F_{\theta}=\left(Q_{\theta}^{-1}\right)^{\circ} Q_{\theta}=$ $-Q_{\theta}^{-1} \dot{Q}_{\theta}$. The Hilbert-Schmidt norm of a $n \times n$ matrix $A$ is defined as $\|A\|_{H S}=\left((1 / n) \operatorname{Tr}\left[A A^{T}\right]\right)^{1 / 2}$. The Gaussian distribution with mean vector $\mu$ and covariance matrix $R$ is denoted by $\mathcal{N}(\mu, R)$.

\section{KNOWN HOST SIGNAL}

When the host signal $s$ is known to the receiver, the linear embedding technique $x=s+p$ (where $x$ is also known to the receiver) provides a benchmark for comparison with the unknown-host case. We have $\mathbb{E}[Y]=G_{\theta} x$ and $\operatorname{Cov}[Y]=$ $\sigma_{w}^{2} Q_{\theta}$. Under regularity conditions, the penalized nonlinear least-squares estimator

$$
\hat{\theta}(y, x)=\operatorname{argmin}_{\theta \in \Theta}\left[\frac{1}{\sigma_{w}^{2}}\left\|H_{\theta} y-x\right\|^{2}+\ln \operatorname{det} Q_{\theta}\right]
$$

\footnotetext{
${ }^{1}$ The paper [1] made less stringent assumptions about $w$.

${ }^{2}$ Here the matrix $Q_{\theta}$ is $\frac{1}{\sigma_{w}^{2}}$ times the matrix $Q_{\theta}$ defined in [1].
} 
has good asymptotic properties as $n \rightarrow \infty$. According to Theorem 1 in [1], $\hat{\theta}(y, x)$ is asymptotically normal:

$$
\sqrt{n}(\hat{\theta}-\theta) \sim \mathcal{N}(0, v(\theta)) \quad \text { as } n \rightarrow \infty
$$

(convergence in distribution), where $v(\theta)=1 / i(\theta, x)$, and

$$
i(\theta, x)=\lim _{n \rightarrow \infty} \frac{1}{n}\left\{\frac{1}{\sigma_{w}^{2}}\left\|F_{\theta} x\right\|^{2}+\operatorname{Tr}\left[F_{\theta}\left(F_{\theta}^{T}+F_{\theta}\right)\right]\right\} .
$$

\section{QUANTIZATION-BASED EMBEDDING AND ESTIMATION}

From here on we assume that the host signal $s$ is unknown to the estimator.

Define a constant $\alpha$, to be optimized later, taking its value in the following range:

$$
\begin{cases}0<\alpha<1 & \text { if } D_{e} \geq \sigma_{w}^{2} \\ 0<\alpha<\frac{2 D_{e}}{D_{e}+\sigma_{w}^{2}}<1 & \text { else. }\end{cases}
$$

Let

$$
D_{v}=(\alpha-1)^{2} D_{e}+\alpha^{2} \sigma_{w}^{2} .
$$

Due to (6), we have $D_{v}<D_{e}$. Also note that $D_{v}$ is minimized by $\alpha^{*}=\frac{D_{e}}{D_{e}+\sigma_{w}^{2}}$.

Next, define a countable set $\mathcal{C}$ of vectors in $\mathbb{R}^{n}$ (called codebook) with the property that $\mathcal{C}$ is simultaneously a good source code for encoding vectors with total distortion $n D_{e}$, and a good channel code for decoding vectors corrupted by noise with total energy $n D_{v}$. A common choice would be a lattice code with nearly spherical cells $[6,7,8]$. The good-source-code property implies that with high probability, $\min _{u \in \mathcal{C}}\|u-\alpha s\|^{2} \leq n D_{e}$. Denote the minimizer by $u=Q(\alpha s)$ (ties are arbitrarily broken). where $Q(\cdot)$ is thought of as a vector quantization operation, and $z=$ $Q(\alpha s)-\alpha s$ as a quantization error. For a good lattice source code, the distribution of $Z$ converges in the Kullback-Leibler sense to $\mathcal{N}\left(0, D_{e} I_{n}\right)$ [9].

Embedding Function. The channel input is constructed as follows:

$$
x=Q(\alpha s)+(1-\alpha) s=\frac{1}{\alpha}(u+(\alpha-1) z) .
$$

Asymptotic Channel Model. The channel output is obtained from (1) and (8):

$$
y=\frac{1}{\alpha} G_{\theta}(u+v)
$$

where $v=(\alpha-1) z+\alpha w$ has zero mean and variance $D_{v}$, and converges in the Kullback-Leibler sense to $\mathcal{N}\left(0, D_{v} I_{n}\right)$. The analysis below is based on the asymptotic channel model (9) with $v \sim \mathcal{N}\left(0, D_{v} I_{n}\right)$.



Fig. 1. Illustration of the host-signal rejection property for parameter estimation. Each tube is a mean-square typical set for $\alpha y$, conditioned on the selected codeword $u$.

\subsection{Estimator}

If $\theta$ is known and $\mathcal{C}$ is a good $D_{v}$-channel code, $u$ can be reliably decoded. Conversely, if $u$ is known, the problem of estimating $\theta$ can be addressed using the methods of [1], and more specifically the penalized nonlinear least-squares estimator

$$
\hat{\theta}(y, u)=\operatorname{argmin}_{\theta \in \Theta}\left[\frac{1}{D_{v}}\left\|\alpha H_{\theta} y-u\right\|^{2}+\ln \operatorname{det} Q_{\theta}\right] .
$$

Under regularity conditions, the normalized estimation error $\sqrt{n}(\hat{\theta}-\theta)$ is asymptotically Gaussian, with mean zero and asymptotic variance $v(\theta)=1 / i(\theta, u)$, where

$$
i(\theta, u)=\lim _{n \rightarrow \infty} \frac{1}{n}\left\{\frac{1}{D_{v}}\left\|F_{\theta} u\right\|^{2}+\operatorname{Tr}\left[F_{\theta}\left(F_{\theta}^{T}+F_{\theta}\right)\right]\right\} .
$$

Let us return to the actual problem in which neither $u$ nor $\theta$ is known to the receiver. Our construction of a reliable estimator of $\theta$ is based on the existence of a universal decoder [5] for $u \in \mathcal{C}$ given $y$ (but not $\theta$ ). Roughly speaking, the codebook $\mathcal{C}$ can be designed so that the "typical sets" $\alpha y$ corresponding to different codewords have statistically negligible overlapping. Each such typical set is represented conceptually as a tube in Fig. 1, where the curve $G_{\theta} u, \theta \in \Theta$ is the axis of the tube indexed by $u \in \mathcal{C}$. Then the codeword $u$ corresponding to $y$ can be reliably identified, and the problem of estimating $\theta$ essentially reduces to the case of known $u$, which was discussed above. We first formally define our estimator, and then state our main theoretical result in the form of Theorem 1.

Estimation Step 1. (Decoding $u \in \mathcal{C}$ ). Choose an arbitrarily small $\eta \in\left(0, D_{e}-D_{v}\right)$ and consider the meansquare typical set

$$
T_{\eta}(u)=\left\{y: \exists \theta \in \Theta:\left\|u-\alpha H_{\theta} y\right\|^{2} \leq n\left(D_{v}+\eta\right)\right\} .
$$


The mean-square typicality decoder for $u$ is defined as

$$
y \in T_{\eta}(u) \Rightarrow \hat{u}(y)=u .
$$

Declare a decoding error if $\nexists u \in \mathcal{C}: y \in T_{\eta}(u)$ or if there is more than one $u$ such that $y \in T_{\eta}(u)$.

Estimation Step 2. Solve the penalized nonlinear leastsquares estimation problem

$$
\hat{\theta}(y)=\operatorname{argmin}_{\theta \in \Theta}\left[\frac{1}{D_{v}}\left\|\alpha H_{\theta} y-\hat{u}(y)\right\|^{2}+\ln \operatorname{det} Q_{\theta}\right] .
$$

Lemma 1. (Consistency of $\hat{u}(y)$.) There exists a codebook $\mathcal{C}$ such that $\operatorname{Pr}_{S}\left[\min _{u \in \mathcal{C}}\|u-\alpha S\|^{2}>n D_{e}\right] \rightarrow 0$ and $\max _{\theta \in \Theta} \operatorname{Pr}_{Y \mid U, \theta}[\hat{U}(Y) \neq U \mid \theta] \rightarrow 0$, as $n \rightarrow \infty$.

Theorem 1. Assume that:

(A1) $G_{\theta}$ is continuously differentiable with respect to $\theta$.

(A2) $G_{\theta} \neq G_{\theta^{\prime}}$ for any $\theta \neq \theta^{\prime}$.

(A3) $\theta$ is an interior point of $\Theta$.

(A4) There exists $M<\infty$ upper bounding the strong norms of $G_{\theta}, H_{\theta}, \dot{G}_{\theta}$ and $\ddot{G}_{\theta}$, for all $\theta$ and $n$.

(A5) The limit (11) exists and is finite and strictly positive. Then $\hat{\theta}(y)$ is an asymptotically unbiased estimator of $\theta$. Moreover, $\hat{\theta}(y)$ is asymptotically normal:

$$
\sqrt{n}(\hat{\theta}-\theta) \sim \mathcal{N}(0, v(\theta)) \quad \text { as } n \rightarrow \infty
$$

(convergence in distribution), where $v(\theta)=1 / i(\theta, u)$.

Outline of the Proof of Theorem 1. The proof relies on the analysis of error probability events of the form

$$
\begin{aligned}
\mathcal{E} & =\left\{y \in \mathbb{R}^{n}:|\hat{\theta}(y)-\theta|<\epsilon\right\}, \\
\mathcal{E}_{u}= & \left\{y \in \mathbb{R}^{n}: \hat{u}(y)=u\right\} \\
& \quad(\text { correct detection of } u) \\
\mathcal{E}_{\not \ell}= & \left\{y \in \mathbb{R}^{n}: \hat{u}(y) \neq u\right\} \\
& \quad \text { (incorrect detection of } u)
\end{aligned}
$$

and on the inequality $\operatorname{Pr}[\mathcal{E}] \leq \operatorname{Pr}\left[\mathcal{E} \cap \mathcal{E}_{u}\right]+\operatorname{Pr}\left[\mathcal{E}_{u}\right]$. The term $\operatorname{Pr}\left[\mathcal{E} \cap \mathcal{E}_{u}\right]$ is analyzed using the same techniques as in Theorem 1 in [1], see (10) and (11). By Lemma 1, $\operatorname{Pr}\left[\mathcal{E}_{\not}\right] \rightarrow$ 0 as $n \rightarrow \infty$.

The proof of the asymptotic normality result proceeds similarly. A critical step in the proof is that the estimation error is bounded under the error event $\mathcal{E}_{\not}$.

Remark \#1. The term $\frac{1}{n D_{v}}\left\|F_{\theta} u\right\|^{2}$ in the expression (11) converges to $\frac{\alpha^{2}}{n D_{v}}\left\|F_{\theta} s\right\|^{2}+\frac{D_{e}}{D_{v}}\left\|F_{\theta}\right\|^{2}$. The first term dominates for $\|s\|^{2}>>n D_{e}$ and is maximized by selecting $\alpha=\alpha_{\max }=\min \left(1, \frac{2 D_{e}}{D_{e}+\sigma_{w}^{2}}\right)$. The asymptotic variance is the same as that for the known-host case in Sec. 3 when $D_{e} \geq \sigma_{w}^{2}$, and slightly worse otherwise.

Remark \#2. The potential role of a source of randomness shared between the embedder and the estimator is to randomize the codebook. In a security application this would help to prevent an adversary from recovering the selected codeword $u$ and requantizing to some other codeword in the vicinity of $u$, prior to implementing a desynchronization attack.

Remark \#3. A necessary condition for nonintersection of the typical tubes $\left\{T_{\eta}(u), u \in \mathcal{C}\right\}$ is that the following quantity be strictly positive:

$$
d_{\min }=\inf _{u \neq u^{\prime} \in \mathcal{C}} \min _{\theta, \theta^{\prime} \in \Theta}\left\|G_{\theta} u-G_{\theta^{\prime}} u^{\prime}\right\| .
$$

\subsection{Example: Scaling Operation}

For a scaling operation, $G_{\theta}=\theta I_{n}, \theta \in\left[\theta_{\min }, \theta_{\max }\right]$, we have $\dot{G}_{\theta}=I_{n}$. Lattice and lattice-coset codebooks are not suitable $\left(d_{\min }=0\right)$ when $\theta_{\max }>2 \theta_{\min }$. Noting that scaling modifies the radial distance of a vector to the origin but not its angle with respect to the coordinate system, we suggest the use of constellation designs to ensure $d_{\min }>0$. This design is illustrated in Fig. 2 in the case of $n=2$. Constellation points have an angular separation of $\Delta \phi$. The quantizer associated with the constellation is a nonseparable polar-coordinate quantizer. The coarseness of the quantizer determines $\Delta \phi$. The signals $s$ considered have Euclidean norm in the finite range $\left[\frac{R_{\min }}{\alpha}, \frac{R_{\max }}{\alpha}\right]$.

As an alternative to the estimator (12) (13), we can also use the following simpler, 3 -step estimator: first, quantize $\arctan y_{2} / y_{1}$ with accuracy $\Delta \phi ;$ second, obtain the location of the corresponding codeword $u$ (constellation decoding); third, let

$$
\tilde{\theta}=\operatorname{argmin}_{\theta}\left\|\frac{\alpha y}{\theta}-u\right\|^{2}=\alpha \frac{\|y\|^{2}}{<y, u>},
$$

and clip $\tilde{\theta}$ to the interval $\left[\theta_{\min }, \theta_{\max }\right]$ to obtain $\hat{\theta}$.

For this constellation, we have $d_{\min } \approx R_{\min } \Delta \phi$.

\section{DISCUSSION}

This paper has addressed both problems of global and local estimation for channel parameters $\theta$. Conditions have been identified under which local-estimation performance (asymptotics) is nearly the same as in the known-host case. Regarding global estimation, a serious difficulty is the potential nonidentifiability of $\theta$ using standard lattice-based codebooks. More work remains to be done to explore the performance and practicality of specialized designs such as constellations.

\section{REFERENCES}

[1] P. Moulin, "Embedded-Signal Design for Channel Parameter Estimation. Part I: Linear Embedding," to appear in IEEE Statistical Signal Processing Workshop, St Louis, MO, Sep. 2003. 


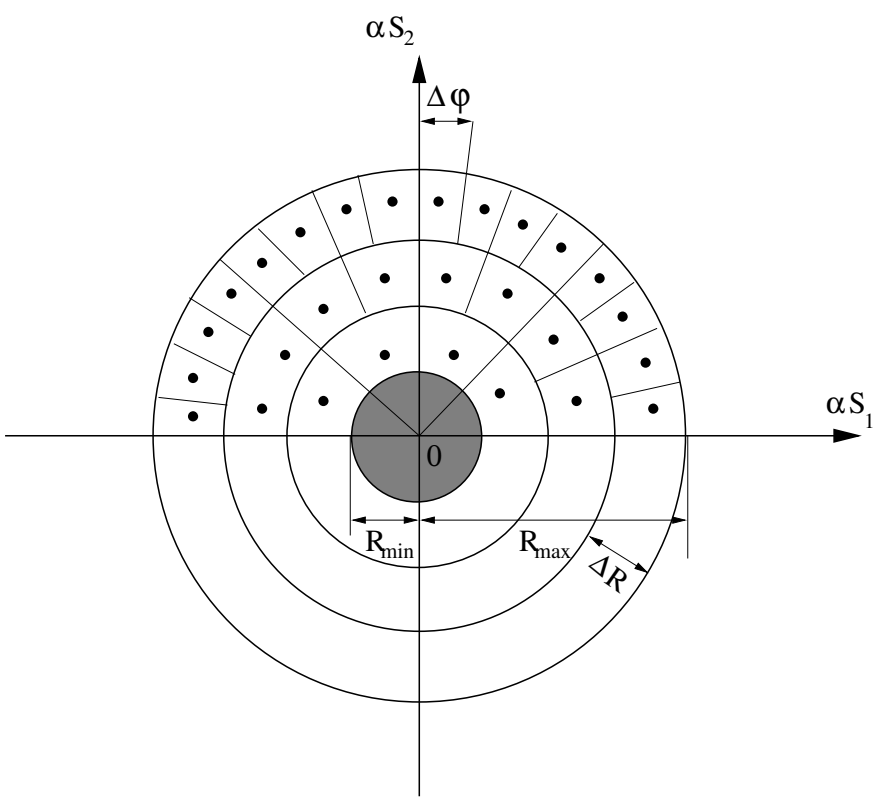

Fig. 2. Signal constellation for estimation of scaling parameter $(n=2$ here $)$.

[2] M. Costa, "Writing on Dirty Paper," IEEE Trans. Info. Thy, Vol. 29, No. 3, pp. 439—441, May 1983.

[3] B. Chen and G. W. Wornell, "Quantization Index Modulation Methods: A Class of Provably Good Methods for Digital Watermarking and Information Embedding," IEEE Trans. Info. Thy, Vol. 47, No. 4, pp. 14231443, May 2001.

[4] T. Liu and P. Moulin, "Error Exponents for Watermarking with Squared-Error Distortion Constraints," Proc. ISIT, p. 190, Yokohama, Japan, July 2003.

[5] A. Lapidoth and P. Narayan, "Reliable Communication Under Channel Uncertainty," IEEE Trans. Info. Thy, Vol. 44, No. 6, pp. 2148-2177, Oct. 1998.

[6] M. Kesal, K. M. Mıhçak, R. Kötter and P. Moulin, "Iteratively Decodable Codes for Watermarking Applications," Proc. 2nd Symposium on Turbo Codes and Related Topics, Brest, France, Sep. 2000.

[7] R. Zamir, S. Shamai (Shitz), and U. Erez, "Nested Linear/Lattice Codes for Structured Multiterminal Binning," IEEE Trans. Info. Thy, Vol. 48, No. 6, pp. 12501276, June 2002.

[8] U. Erez and R. Zamir, "Lattices which are Good for (Almost) Everything," preprint, June 2003.

[9] R. Zamir and M. Feder, "On Lattice Quantization Noise," IEEE Trans. Info. Thy, Vol. 42, No. 4, pp. 1152-1159, July 1996. 